\title{
The durability of oral diabetic medications: Time to A1c baseline and a review of common oral medications used by the primary care provider
}

\author{
Lavanya Cherukuri, Michael S. Smith, and John A. Tayek* \\ Department of Internal Medicine, Los Angeles Bio-Medical Research Institute (LABioMed), Harbor-UCLA Medical Center \\ David Geffen School of Medicine at UCLA, USA
}

*Correspondence to: John A. Tayek, 1000 W. Carson Street, Box 428, Torrance, CA, 90509, Phone: 310-222-1237; Fax: 310-320-8459; E-mail: jtayek@dhs.lacounty.gov

Received: June 30, 2018; Accepted: July 07, 2018; Published: July 08, 2018;

\begin{abstract}
Introduction: Cost of generic medications has risen more in the past few years than any other time in history. While medical insurance covers much of these costs, health care professionals can better provide medications that have the longest duration of action when compared to placebo-treated controls. This will save health care costs and improve prescribing accuracy.

Methods: Papers in PubMed were identified with keywords placebo. The study must be at least 2 years in length to evaluate the change in A1c over time. The primary endpoint was time to A1c neutrality (return of A1c to baseline at a maximum dose of single oral agent). A medication would be considered at neutrality if the $95 \%$ CI crossed baseline. Time to neutrality was averaged for each medication within the class and each summarized for class effect.

Results: Effective therapy for the DPP-4 and sulfonylurea classes of medications are 3-4 years as compared to a 5-year time to A1c neutrality for metformin usage. In comparison, the projected time to A1c neutrality was approximately 6-8 years for rosiglitazone and pioglitazone. While only a few studies have been published in the SGLT-2 class of medication, the time to A1c neutrality was also 6-8 years with Canagliflozin and full dosage of Empagliflozin.

Conclusion: Metformin appears to have a 5-year duration of effect before the A1c returns to baseline. The sulfonylureas and DPP-4 inhibitors class of medications have one of the shortest durability which ranges between 3.3 to 4.4 years. In contrast, the SGLT-2 class of medication and the TZD class of medications has a projected time to A1c neutrality from 6-8 years. Diabetic duration of therapy as compared to placebo should be listed with those medications tested so the provider can choose wisely.
\end{abstract}

\section{Introduction}

ADA and AACE suggest that metformin be the first line medication for type 2 diabetes mellitus with many choices for the second line agent (ADA 2018, AACE 2016). Primary care health care professionals would benefit from understanding the potential durability of the diabetic medications to help improve compliance and reduce cost. Historically, sulfonylureas have been added second to metformin, fortunately, over the last 15 years, many combination agents have been developed that include metformin as one of the combo medications. So, the decision to choose the best second agent should be based on the evidence of safety and durability provided.

Limited studies have evaluated the long-term durability of a single diabetic agent on A1c control. The ADOPT trial used monotherapy with Metformin, Glyburide or Rosiglitazone and evaluated A1c changes over a 5 -year period. Since the potential risk of rosiglitazone causing CV disease in 2008, the FDA has regulated that all diabetic medications have a CV trial to demonstrate safety. Prior to this regulation by the FDA, the ADOPT trial was a landmark study to evaluate the durability of single diabetic agents [1]. Since the 2008 FDA requirement, all oral diabetic medications have been evaluated with a major endpoint being cardiovascular safety which requires large sample size and longer duration of therapy. Oral diabetic meds were evaluated in randomized, single agent, placebo-controlled clinical cardiovascular trials (with one exception being the TECOS trial) were used to also evaluate the durability of these oral medications based on baseline A1c nadir, return to baseline and compared to placebo treatment.

\section{Methods}

Based on the approach of the ADOPT [1] trial, where monotherapy was tried with three separate agents over an extended period of time (minimum 2 years), the study was designed to evaluate newer diabetic medications when given in placebo driven clinical trials for a minimum of 2 years. Trials with the several class of medications have recently been completed and the data summarized [2-9].

The ADOPT trial demonstrated what monotherapy with metformin, glyburide or rosiglitazone will reduce Alc levels over a 5 -year period. Since DM-2 is considered a progressive disease with slow loss of the beta cell function, the waning effect of each medication was documented and projected time to A1c neutrality was documented over the 5-year study [1]. 
John A. Tayek (2018) The durability of oral diabetic medications: Time to A1c baseline and a review of common oral medications used by the primary care provider

The purpose of the trial was to clarify the duration of action of the more commonly used classes of medications. Traditionally the choice of medications has been limited and now there are over 20 combination oral medications for use in the treatment of type 2 diabetes. If the provider appreciates the duration of action of these medications then more appropriate choices can be made which should reduce the cost of medications and improve overall diabetic control.

Table 1. Duration of Alc Effect over Time vs Placebo (Return to Baseline)

\section{Results}

Three of the five FDA approved DPP-4 medications have had CV safety tested in a double-blind and placebo-controlled design.

The first two agents' saxagliptin (2) and alogliptin (8) were published on the same day $(9 / 2 / 13)$ and they demonstrated a drop in A1c that was not significantly lower than baseline at the end of study. The Sitagliptin study showed a significant drop in A1c at 12 weeks but the difference from baseline was not significant after 34 weeks nor at the end of the 4-year study (Table 1 and Figure 1).

\begin{tabular}{|c|c|c|c|c|c|c|}
\hline NAME & $\begin{array}{l}\text { Dose (mg) } \\
\text { Total Daily }\end{array}$ & $\begin{array}{c}\text { Baseline A1c } \\
(\%)\end{array}$ & Early (Nadir) & Mid level & End of Study & $\begin{array}{l}\text { Time to A1c } \\
\text { Neutrality }\end{array}$ \\
\hline Alogliptin (EXAMINE) & 30 & 8.0 & $-0.7 / 12 w^{*}$ & & $-0.36 / 3 y r s^{*}$ & $3.3 \mathrm{yrs}$ \\
\hline Sitagliptin (Green) & 100 & 7.2 & $-0.3 / 4 \mathrm{w}(\mathrm{NS})$ & $-0.2 / 34 \mathrm{w}(\mathrm{NS})$ & $-0.1 \% / 4 \mathrm{yrs}$ & $4.0 \mathrm{yrs}$ \\
\hline Saxagliptin (Scirica) & 5 & 8.0 & $-0.5 / 104 w$ & & $-0 \% / 4 \mathrm{yrs}$ & $4.0 \mathrm{yrs}$ \\
\hline \multicolumn{7}{|r|}{ Gliptins: (3-4 yrs) } \\
\hline Glipizide (Feinglos) & 2.5 & 8.0 & $-0.5 / 14 w$ & & & $3.0 \mathrm{yrs}$ \\
\hline Glyburide (ADOPT) & 20 & 7.3 & $-0.90 / 12 w$ & & & $3.7 \mathrm{yrs}$ \\
\hline \multicolumn{7}{|r|}{ Sulfas: (3-4 yrs) } \\
\hline Metformin (ADOPT) & 2500 & 7.3 & $-0.60 / 52 \mathrm{w}$ & & & $5.0 \mathrm{yrs}$ \\
\hline \multicolumn{7}{|c|}{ Metformin: (5 yrs) } \\
\hline Rosiglitazone (ADOPT) & $4-8$ & 7.3 & $-0.67 / 104 w$ & & & $8.0 \mathrm{yrs}$ \\
\hline Pioglitazone (PROACTIVE) & 45 & 7.8 & & & $-0.80 / 3 \mathrm{yrs}$ & $6.0 \mathrm{yrs}$ \\
\hline \multicolumn{7}{|r|}{ TZDs: (6-8 yrs) } \\
\hline Empagliflozin (EMPA) & 10 & 8.1 & $-0.54 / 12 \mathrm{w}$ & $-0.42 / 94 w$ & $-0.36 / 206 \mathrm{w}$ & $5.0 \mathrm{yrs}$ \\
\hline “ & 25 & 8.1 & $-0.60 / 12 \mathrm{w}$ & $-0.47 / 94 w$ & $-0.42 / 206 w$ & $8.0 \mathrm{yrs}$ \\
\hline Canaglifozin(CANVAS) & $100 / 300$ & 8.2 & $-0.70 / 26 \mathrm{w}$ & $-0.40 / 156 w$ & $-0.20 / 286 w$ & $7.0 \mathrm{yrs}$ \\
\hline
\end{tabular}

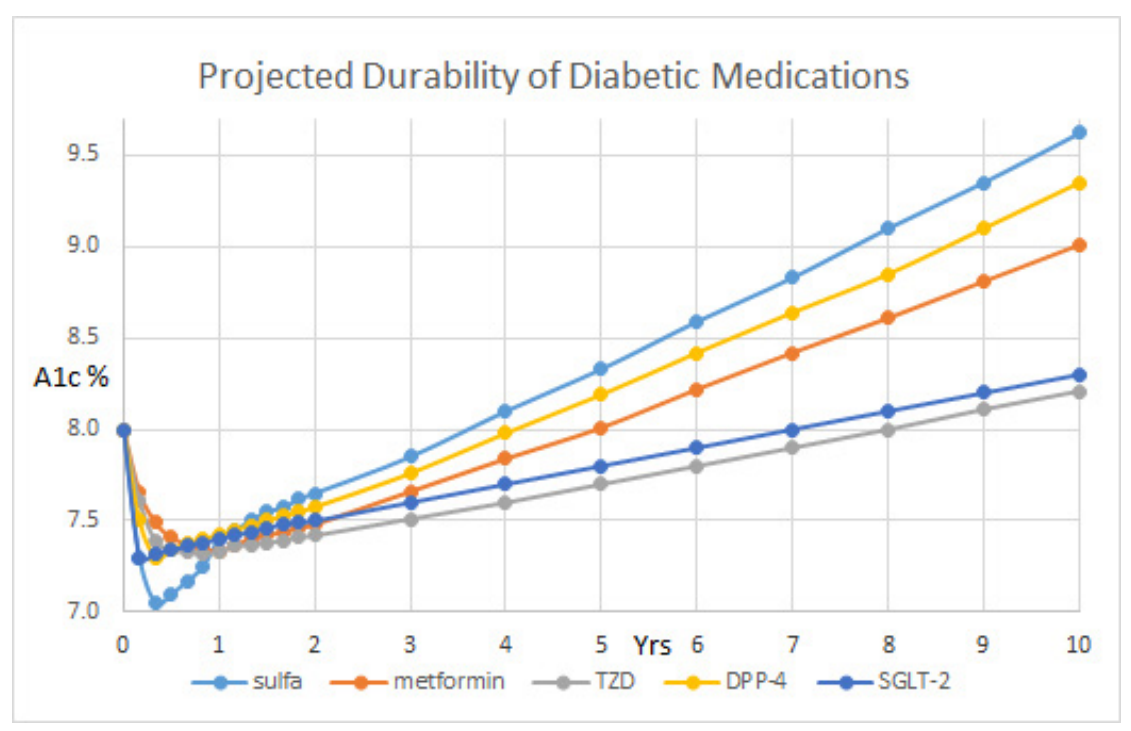

Figure 1. Projected durability of diabetic medications. 
The first of three DPP-4 clinical trials demonstrated that saxagliptin use for 2.1 years had no CV benefit and it reduced A1c from 8.0 to $7.7 \%$ which was $0.2 \%$ less than placebo [2]. Unfortunately, the SAVOR trial demonstrated that more saxagliptin-treated patients developed heart failure than placebo-treated patients (3.5\% vs $2.8 \%$; $\mathrm{NNH}=143$ ). This potential risk is listed with the FDA for health care professionals to evaluate and discuss with their patients [8]. Alogliptin treatment for 3 years dropped A1c from $8.0 \%$ to $7.67 \%$ which was $0.36 \%$ less than the placebo at the end of the 3 years but there was no cardiovascular benefit observed [3]. Sitagliptin was given for 3 years and it had no CV benefit and it reduced the A1c from $7.3 \%$ to $7.1 \%$ which was $0.29 \%$ less than the placebo at the end of the 3 years [4].

In comparison, monotherapy was provided with glyburide over 5 years and the results after 3 years demonstrated a significant drop in A1c from 7.3\% to 7.1\% (1). Low dose glipizide XL has 0.5\% drop in A1c which nadirs, like glyburide at 12-14 weeks. Glipizide appears to have a shorter duration of action as it is projected to return to baseline at approximately 1 year. Likewise, metformin was given a monotherapy and the A1c dropped from $7.3 \%$ to $6.9 \%$ at 3 years. Rosiglitazone was also given and it dropped the A1c from $7.3 \%$ to $6.85 \%$ at 3 years [1]. The A1c returned to its baseline of $7.3 \%$ after 3.75 years of starting glyburide and after 5 -years after starting metformin.

The TZD class of medications appears to provide for a longer duration of action. Interestingly, the Alc did not return to normal after 5 years of starting rosiglitazone as it remained significantly reduced at $7.1 \%$. If you extend linear trend for A1c, it appears to take approximately 8 years for the A1c to return it to baseline of $7.3 \%$. In the PROACTIVE trial, pioglitazone treatment for 3 years significantly reduced A $1 \mathrm{c}$ from $7.8 \%$ to $7.0 \%$ at the end of 3 years [5]. The placebotreated patients drop their Alc by $0.3 \%$ at the end of study which showed no difference from baseline.

Pioglitazone treatment in the PROACTIVE trial demonstrated a $0.8 \%$ drop in $\mathrm{A} 1 \mathrm{c}$ at the end of the 3 years study with a predicted durability of 6.0 years [5]. In contrast, the Empagliflozin data demonstrated a $0.4 \%$ drop in A1c at the end of the 4-year study which projected to a 5-8 years durability of this therapy depend on the dose studied [6].

It would appear that the effective therapy for the DPP-4 class is approximately 3-4 years as compared to 5 years for the time for the Alc measurement to return the baseline level for metformin. The 5 year duration of action for metformin was confirmed in the Diabetes Prevention Trial where FBG was $106 \mathrm{mg} / \mathrm{dl}$ at baseline and returned to $106 \mathrm{mg} / \mathrm{dl}$ after 5 years [17]. In the placebo arm the FBG increased from 106 to $112 \mathrm{mg} / \mathrm{dl}$ over the 5 -year period. In the 15 year follow up report the FBG increased to $117 \mathrm{mg} / \mathrm{dl}$ in the metformin arm, which is smaller then the projected change in Alc over time in Fig 1. In contrast, the projected time to A1c baseline was approximately 6-8 years for rosiglitazone and pioglitazone. While only two large studies have been published in the SGLT-2 class of medication, it appears that the time to Alc neutrality was seen Canagliflozin was 7 years [7] and with Empagliflozin an estimated 5 years with a low dose and 8 years with high dose [6].

\section{Discussion}

The natural history of type 2 diabetes mellitus appears to be due to persistent loss of the beta-cell function over time. While there can be an increase in insulin resistance that can occur with aging or weight gain, the majority of the waning effect of diabetic medications are likely due to the natural loss of insulin secretion. Why this rate is different with different medication is unknown.

We know that fasting insulin levels are increased with glyburide treatment and remain neutral with both metformin and DPP-4 inhibitors. The mechanism of the DPP-4 inhibitors is to increase incretin levels (GLP-1 and GIP), which inhibits glucagon release, which in turn increases insulin secretion, decreases gastric emptying and decreases blood glucose levels. While metformin has a neutral effect, the administration of TZD medication and that of the SGLT-2 medication are associated with a significant reduction in the fasting insulin levels [10]. The reduction in the insulin levels is unlikely to play a major role in the durability because of the failure of Origins trial to delay the onset of DM-2, where basal insulin was administered for 5 years [11]. So, TZD and SGLT-2 class appear to have a direct drug effect on the beta cell and/or other pathways of insulin resistance. We know that liver fat, beta cell fat and selective muscle fat is reduced with TZD treatment [10]. Likewise, weight loss seen with the SGLT-2 inhibitors can be associated with similar changes in these tissues that could both reduce insulin resistance and increase insulin secretion.

Diabetes appears to be a progressive disease process with slow but consistent loss of control over 6 to 12 months of therapy in many patients. It is well known that the use of TZD reduces carotid intima thickness as compared to glimepiride [10], it reduces liver fat content by $54 \%$ [12] and improves insulin resistance and beta cell function [10]. Metformin prevents the production of glucose in both the liver and kidney which may reduce the insulin requirements associated with hormonal or metabolic factors that would otherwise rise insulin secretion.

This raises two interesting points: durability of each class of medications and potential mechanism of action of these medications. The best durability appears to be in the TZD and SGLT- 2 classes which appears... to have a projected effect on A1c of greater than 6-8 years while metformin has limited benefit of approximately 5 -years before the A1c returns to baseline [1]. The shortest durability is seen in the DPP-4 medications, which have 3-4 years projected time to Alc neutrality. Therefore, the time for A1c neutrality appears to be the shortest for the DPP- 4 and sulfa classes of medications.

What to do about the waning effect of several classes of mediations: The current answer is unknown, but if the effective treatment benefit of a DPP-4 medication is approximately 3-4 years and metformin has 5 years, it seems clear that metformin has both durability and cost savings. The combinations agents with both metformin and DPP-4s may misleading health care professionals to its durability since metformin is likely doing the majority of the treatment effect. Interestingly, the use of rosiglitazone, the medication that caused the introduction of the FDA for Cardiovascular Outcome trials to confirm $\mathrm{CV}$ neutrality has been shown to have one of the two longest duration of benefit for $\mathrm{A} 1 \mathrm{c}$ reduction over time. 
Caution about the use of both Rosiglitazone and Pioglitazone exists in the medical literature. Rosiglitazone was put on restricted usage requiring REMS after Dr. Steve Nisson published his analysis of the increase CV risk that appears to be associated with rosiglitazone [15]. This REMS requirement for rosiglitazone was withdrawn by the FDA after publication of the RECORD trial where there was no CV risk associated with rosiglitazone [16]. This class of medications is contraindicated in CHF stage 3 or 4 . They are known to cause intravascular fluid retention and also to cause lower extremity swelling which is likely due to pre-adipocyte differentiation into adipocytes. This class of medications lowers fasting insulin levels and also lower IGF-1 concentrations which likely explains the loss of bone mass over time. The TZD class of medications should be avoided in patients with significant osteoporosis.

In comparison to the downsides of using the TZD class of medication, there is growing evidence suggests that pioglitazone treatment has shown a $51 \%$ resolution of NASH and reduces fibrosis [13]. In addition, pioglitazone has been shown to help reduce recurrent CVA in diabetics [5] and pre-diabetics [14]. Similarly, the SGLT-2 medication Empagliflozin has been approved by the FDA to reduce $\mathrm{CV}$ risk and it has a projected long-term reduction in A1c levels. Based on the duration of action, potential CV risk reduction, this medication would be an excellent choice for diabetic patients at risk for CV events. Likewise, pioglitazone may be a wise choice for pre-diabetic or diabetic patients who have had a recent CVA event or have F3-F4 fibrosis or proven NASH. Additional CV outcome trials with newer diabetic medications will also provide the provider with duration of effect estimate which may help improve compliance and reduce health care costs.

In conclusion, the primary care provider should consider the durability of treatment in their treatment decision after starting metformin. The A1c durability of each agent should be included in treatment guidelines for ACP, ADA, AACE and European Diabetic Guidelines. In the time of abundant oral diabetic medications, effort should be used to educate health care professionals on the duration of action as well as potential non-diabetic risk and benefits of diabetic agents. More studies on the comprehensive effective long-term, placebo-controlled monotherapy treatment trials will allow the health care professionals can choose wisely for their patients.

In summary, the duration of A1c effect was 5 years for metformin and it appears to be a better choice over a sulfa and DPP-4 class of medications. A longer duration of action was seen with the SGLT-2 and TZD class of medications. These both have a long duration of action (6-8 yrs), and with their low cost may be considered in patients who would benefit from their use.

With the recent adoption by the ACP (American College of Physicians) that target A1c of $<8 \%$ is "at goal" for diabetic patients, then metformin should provide 5 years of acceptable diabetic control if stating at an A1c of 8\%. Our data would suggest that mono-therapy with a SGLT-2 inhibitor or PPAR-gamma agonist (TZD) would keep someone at goal for approximately 7-8 years if the starting A1c was $8 \%$. Using this stepwise triple therapy approach, it may be possible for a diabetic patient to maintain their A1c at goal for approximately 20 years.
Acknowledgement: The NIH Clinical Investigator Award KO8DK02083 and MO1-RR-00425 supported this grant

\section{References}

1. Kahn SE, Haffner SM, Heise MA, Herman WH, Holman RR, et al. (2007) Glycemic durability of rosiglitazone, metformin, or glyburide monotherapy. $N$ Engl J Med 355: 2427-2443. [crossref]

2. Scirica BM, Bhatt DL, Braunwald E, Steg PG, Davidson J, et al. (2013) Saxagliptin and cardiovascular outcomes in patients with type 2 diabetes mellitus. $N$ Engl J Med 369: 1317-1326. [crossref]

3. White WB, Cannon CP, Heller SR, Nissen SE, Bergenstal RM, et al. (2013) Alogliptin after acute coronary syndrome in patients with type 2 diabetes. $N$ Engl $J$ Med 369: 1327-1335. [crossref]

4. Green JB, Bethel MA, Armstrong PW, Buse JB, Engel SS, et al. (2015) Effect of Sitagliptin on Cardiovascular Outcomes in Type 2 Diabetes. $N$ Engl J Med 373: 232-242. [crossref]

5. Dormandy JA1, Charbonnel B, Eckland DJ, Erdmann E, Massi-Benedetti M, et al. (2005) PROactive Investigators. Secondary prevention of macrovascular events in patients with type 2 diabetes in the PROactive Study (PROspective pioglitAzone Clinical Trial In macroVascular Events): a randomized controlled trial. Lancet 366 : 1279-1289. [crossref]

6. Zinman B, Wanner C, Lachin JM, Fitchett D, Bluhmki E, et al. (2015) Empagliflozin, cardiovascular outcomes, and mortality in type 2 diabetes. $N$ Engl J Med 373 2117-2128. [crossref]

7. Mahaffey KW, Neal B, Perkovic V, de Zeeuw D, Fulcher G, et al. (2017) Canagliflozin for primary and secondary prevention of cardiovascular events: Results from the CANVAS program. Circulation 137: 323-334.

8. Kongwatcharapong J, Dilokthornsakul P, Nathisuwan S, Phrommintikul A, Chaiyakunapruk N (2016) Effect of dipeptidyl peptidase-4 inhibitors on heart failure: A meta-analysis of randomized clinical trials. Int J Cardiol 211: 88-95. [crossref]

9. Feinglos M, Dailey G, Cefalu W, Osei K, Tayek J, et al. (2005) Effect of glycemic control of the addition of $2.5 \mathrm{mg}$ glipizide GITS to metformin in patients with type 2 diabetes. DM Res Clin Prac 68: 167-182.

10. Langerfeld MR, Forst T, Hohberg C, et al. (2005) Pioglitazone decreases carotid intima-media thickness independently of glycemic control in patients with type 2 diabetes mellitus: Results from a controlled randomized study. Circulation 17: 2525-2531. [crossref]

11. Gerstein HC, Bosch J, Dagenais GR, Díaz R, Jung H, et al. (ORIGINS Investigators) (2012) Basal insulin and cardiovascular and other outcomes in dysglycemia. $N$ Eng J Med 367: 319-328. [crossref]

12. Belfort R, Harrison SA, Brown K, Darland C, Finch J, Hardies J (2006) A placebocontrolled trial of pioglitazone in subjects with nonalcoholic steatohepatitis. $N$ Eng J Med 355: 2297-307. [crossref]

13. Cusi K, Orsak B, Bril F, Lomonaco R, Hecht J, et al. (2016) Long-term pioglitazone treatment for patients with nonalcoholic steatohepatitis and prediabetes or type 2 diabetes mellitus: A randomized trial. Ann Intern Med 305-315. [crossref]

14. Kernan WN, Viscoli CM, Furie KL, Young LH, Inzucchi SE, et al. (2016) Pioglitazone after ischemic stroke or transient ischemic attack. $N$ Engl J Med 374: 1321-1331. [crossref]

15. Nissen SE, Wolski K (2007) Effect of rosiglitazone on the risk of myocardial infarction and death from cardiovascular causes. $N$ Engl J Med 356: 2457-71. [crossref]

16. Mahaffery KW, Kafley G, Dickerson S, Burns S, Tourt-Uhlig S, et al. (2013) Results of reevaluation of cardiovascular outcomes in the RECORD trial. Am Heart $J$ 166: 240-249. [crossref]

17. Nathan DM et el (Diabetes Prevention Program Research Group), Long term effects of lifestyle intervention or metformin on diabetes development and microvascular complications over 15-year follow-up: the Diabetes Prevention Program Outcome Study. (2015) Lancet Diabetes Endocrinol 3: 866-875.

\section{Citation:}

Lavanya Cherukuri, Michael S. Smith, John A. Tayek (2018) The durability of oral diabetic medications: Time to A1c baseline and a review of common oral medications used by the primary care provider. Endocrinol Diabetes Metab J Volume 2(3): 1-4 